\title{
A Multi-scale Perspective of Interfaces-dominated Mechanical Behavior
}

\author{
Jian Wang
}

Interfaces in heterophase and homophase crystalline solids are important due to their role in material properties such as strength, fracture, work hardening, and damage evolution under irradiation and shock. The role of interfaces in plastic deformation of metals encompasses interfaces acting as sources, sinks, barriers, and storage sites. Correspondingly, many deformation modes related to these roles can be activated from interfaces. These roles become crucial as grain size is considered at the nanoscale. In such interfacedominant materials, the properties of the interface control all defect-related processes, and those of the constituents play only a secondary role.

A solid fundamental understanding of interfacial structure-property relationships would open the way to tuning the interface structures in interface-dominant materials to meet the demands of future applications. To succeed in this effort, the key challenges are understanding the role of interface structures and properties during deformation and developing a design principle to enhance or suppress some of these roles through control of interfaces. In this regard, four papers included in this issue involve three aspects in understanding the roles of interface from atomic-scale to meso-scale.

The first aspect is on the formation and role of twin boundaries. Twin boundaries are high symmetry interfaces in crystalline solids. Nevertheless, as interfaces, twin boundaries interrupt dislocation glide due to the discontinuity of slip systems across them and affect the mechanical properties of materials. To take full advantage of this effect, it is desirable to introduce twin boundaries in a controllable fashion. For one-dimensional nanowires,
Hanchen Huang at the University of Connecticut reviews various ways of introducing twin boundaries, with particular emphasis on the new approach of combining mechanical torsion and local heating. The role of twin boundaries during mechanical deformation, particularly using in-situ high resolution transmission electron microscopy, has been explored by N. Li et al. at Los Alamos National Laboratory. Their recent studies have provided new insights on the deformation behavior of nanotwins. Two unit processes are observed for the first time: stress-induced migration of $\Sigma 3\{112\}$ incoherent twin boundary that leads to zero-strain detwinning of nanotwins, and multiplication of twinning dislocation at $\Sigma 3\{111\}$ coherent twin boundary.

The second aspect is on the heterogeneous plastic deformation related to grain boundaries. The paper by Y. Yang et al. describes progress in understanding the processes of heterogeneous plastic deformation and facture initiation in titanium by coupling experimental measurements with crystal plasticity finite element (CPFE) simulations. The authors measured the dislocation activity by using techniques associated with atomic force microscopy, confocal microscopy, three-dimensional $\mathrm{x}$ ray diffraction, and nano-indentation, and developed the CPFE model with the guidance of these measurements. The developed CPFE model successfully predicted most types of active dislocation systems within grains at the correct magnitudes, but the simulation of spatial distribution of strain was not always similar to experimental observations. This indicates that a major challenge for model development is to effectively predict conditions where slip transfer occurs, and where geomet- rically necessary dislocations accumulate.

The third aspect is on the role of interface under shock conditions. Shock loading is an extreme mechanical condition that causes the formation of dislocation cells/walls, twins, phase transformations, sub-boundaries, shear bands, recrystallized grains, etc. as observed in a TEM. While such observations could not provide atomic-scale information by today's experimental techniques. In this regard, atomic-scale simulations are a valuable tool for studying the energetic and kinetics of defect nucleation, reactions, and interactions. The paper by N. Gupta et al. reviews the effect of interface structure on the spallation process of multipleinterface composites. In contrast to single crystal face-centered cubic metals, the authors found that while the interface acts as an organized source of partial dislocation loops, structural features that developed during wave propagation causes failure at the interface. However, the presence of the amorphous structure "frustrated" dislocation nucleation behavior that greatly speeded the void formation.

In a summary,a multi-scale perspective of interface-dominated mechanical behavior is essential. Atomic studies using atomistic simulations and electron microscopy, correlation studies of interfaces and local deformation using electron backscatter diffraction, and the development of theoretical and modeling models at different lengthscale incorporating interface physics are recommended.

Jian Wang is a technical staff member at Los Alamos National Laboratory and is the advisor to JOM from the Nanomechanical Materials Behavior Committee of the Materials Processing \& Manufacturing Division of TMS. 